\title{
Cross-cultural adaptation and preliminary validation of a zygosity determination questionnaire for twins in Sri Lanka
}

\author{
A Sumathipala ${ }^{1}, \mathrm{~N}$ De Silva ${ }^{2}, \mathrm{SH}$ Siribaddana ${ }^{3}, \mathrm{MRN}$ Abeysingha ${ }^{4}$ and DJS Fernando ${ }^{5}$ \\ ${ }^{1}$ Section of Epidemiology and General Practice, Institute of Psychiatry, Kings College, University of London, UK \\ ${ }^{2}$ National Twin Registry Project, Colombo \\ ${ }^{3}$ Sri Jayewardenepura General Hospital, Nugegoda \\ ${ }^{4}$ Epidemiology Unit, Colombo \\ ${ }^{5}$ Department of Medicine, Faculty of Medical Sciences, Sri Jayewardenepura University, Nugegoda, Sri Lanka
}

\begin{abstract}
We report the process of adaptation into Sinhala of a questionnaire given to mothers of twins to determine zygosity. Adaptation and validation was carried out in three stages. Firstly, we used a nominal group to translate the English version and to assess the extent of agreement (consensus measurement) on the appropriateness of the translation and resolve disagreement (consensus development). Secondly we used a qualitative interview with 25 mothers of twins. The three main stems of the translated questionnaire were used as a semi-structured interview, and the responses noted verbatim. These were categorised and analysed, and the translated full questionnaire was then presented as closed questions with fixed choice responses. The categorised responses generated during the qualitative interview were compared with the responses to the fixed choices in the full questionnaire. The third stage was the appraisal of the questionnaire by 17 bilingual parents of twins. The source and translated version of the questionnaire were given to them at least 3 days apart. The responses were rated and the total scores were computed to determine the zygosity. This step was carried out to measure the validity and reliability of the Sinhala version. A perfect correlation between the original and adapted version was obtained, with a kappa of 1 . The results suggest that the Sinhala version of the questionnaire is conceptually equival ent to the original questionnaire. Comparison of the zygosity determination by using this adapted questionnaire with results from analysis of genetic markers on Sri Lankan twins is needed for final validation of the translated questionnaire. Twin Research (2000) 3, 205-212.
\end{abstract}

Keywords: nominal group, consensus measurement, consensus development, Sinhala, semi-structured interview, qualitative research, probe technique, pre-testing

\section{Introduction}

Zygosity determination is an essential component of twin research. The most accurate method for zygosity determination is the use of genetic markers. ${ }^{1}$ However, there is a high correlation between genetic methods and questionnaire methods in studies carried out in the US, UK and Scandinavia using large twin samples. ${ }^{2-5}$ Questionnaire methods are preferable for developing countries as genetic methods are expensive. Most questionnaires are to be answered by twins themselves, ${ }^{1}$ and zygosity determination based on the information obtained from parents of twins is comparatively limited. ${ }^{6}$ Also, existing questionnaires were developed mainly in the developed

Correspondence: A Sumathipala, Section of Epidemiology and General Practice, Institute of Psychiatry, Kings College, University of London, Denmark Hill, London, SE5 8AF, UK.

Tel: + + 941875354 ; Fax: + + 941578496 ;

E-mail: spjuats@op.bpmf.ac.uk world. The Sri Lankan twin registry has a higher ascertainment for younger twins and adaptation of a suitable questionnaire was required. ${ }^{7}$ We used the zygosity determination questionnaire for mothers of twins developed by Ooki, Yamada and Asaka, ${ }^{1}$ for translation and adaptation into Sinhal a to use among the Sinhala-speaking majority of the population in Sri Lanka.

Valid use of instruments across cultures requires a careful adaptation process that goes beyond mere language translation. ${ }^{8}$ Kleinman criticised medical research often proceeding as if translations were a nuisance that has to be handled quickly. ${ }^{9}$ There are certain issues when working with translations, namely content validity (contents of the instrument should be culturally relevant), semantic validity (words used in the original and the translated versions have the same meaning), technical validity (similar effect to be achieved by the measuring technique in different cultures), criterion validity (measures whether responses to similar items relate to the same normative concept in two cultures) and 
conceptual validity (requires that responses to an interview relate to a theoretical construct within the culture). ${ }^{10,11}$ Idiomatic equival ence is another problem in translations and was particularly relevant to this questionnaire. Since idioms and colloquialism are rarely translatable, equivalent expressions have to be found or items have to be substituted. ${ }^{12}$

\section{Current approaches}

The current practices of translation include backtranslation, decentering, bilingual approach, and the committee approach. ${ }^{12,13}$ In the back-translation method, translation is carried out while trying to change as little as possible. ${ }^{13}$ This procedure yields three versions of the instrument, one in the original language, one translated and a third back-translated version to the original language. A comparison of the two versions in the original language provides a basis for assessing the translation for its appropriateness. $^{14}$ Decentering, on the other hand, does not require that the final version of the instrument is close in form or content to the original. ${ }^{13}$ It requires that the conceptual domain of the instrument, rather than the structure of any particular language, determines how the final product will look. In the bilingual approach, the original and the translated versions are given to a group of bilingual people and compared for similarity in the responses. In the committee approach, a committee is used for translation as well as for assessing the correctness of the translation. Sumathipala and Murray ${ }^{15}$ used a qualitative method; a nominal group for translation and consensus generation. That revealed several advantages over conventional methods. In this translation and adaptation attempt, we extended that process by combinations of two more steps mentioned in the methods section for the adaptation process.

\section{Zygosity questionnaire for twin mothers}

The source questionnaire by Yamada and Asaka ${ }^{1}$ consisted of three questions concerning the degree of similarity of twins at one year of age; whether they were confused with one another and if so by whom. According to the degree of similarity of twins, points were allocated from 1-3 for answers. If the sum of points was 3-6, the twins were considered $M Z$ and if the sum was $7-10$ they were considered DZ. At this cut of score, the questionnaire has $90 \%$ accuracy in discriminating $M Z$ and $D Z$ twins in comparison to genetic markers.

\section{Methods}

\section{Stage 1}

1. We convened a nominal group for translation and consensus generation. By this process we translated the introduction, three main stems and nine choices of responses. For details of this method, please see Sumathipala and Murray. ${ }^{15}$

Participants Our panel consisted of a total of nine individuals enrolled on the basis of fluency in both English and Sinhala. On the grounds of expertise, the first author participated as one of the panellists and facilitated the group. ${ }^{16,17}$

Source instrument for translation The source questionnaire is by Yamada and Asaka ${ }^{1}$

Procedure (Figure1) Each participant was provided with the source questionnaire for translation. Translations were done individually.

1. The principles of this method ${ }^{16-19}$ were presented by the first author (AS) and a discussion followed to clarify the process.

2. All participants recorded their own translations of each question on separate flip charts. This was done in order to rate each translation individually without any comparison with others. Initials of the participant were noted on the back of the flip chart. Only one item at a time of the original English version of the questionnaire and its corresponding nine translations were taken up for evaluation and consensus generation.

3. During the first round similar translations were grouped together.

4. Each participant privately rated the appropriateness of the translation on a scale of 1 to 9 on the rating sheet prepared for the first $^{6,17,18}$ (see Table 1 for details). They were asked to decide whether each translation represented the idea conveyed by the original item in English. We were concerned about the total meaning conveyed by the sentence rather than a direct word-for-word translation. Therefore linguistic equivalence was less important than conceptual and semantic equival ence. No discussion took place during this round. Each translation was rated individually without discussion. Rating sheets were collected at the end. 

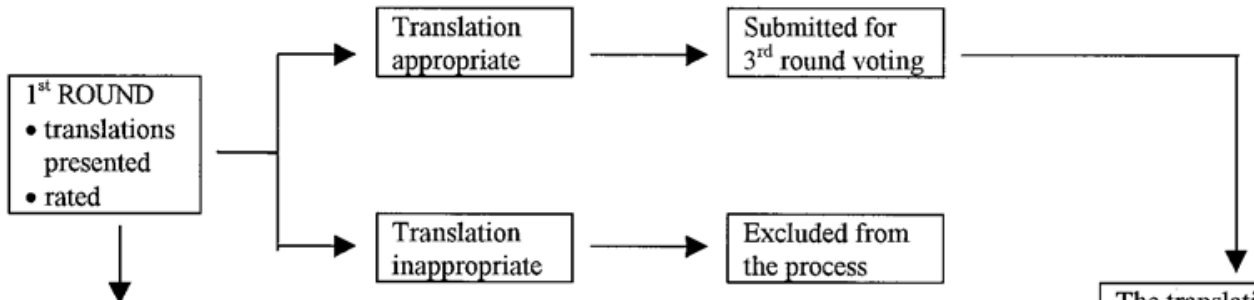

Failed to

reach a

consensus

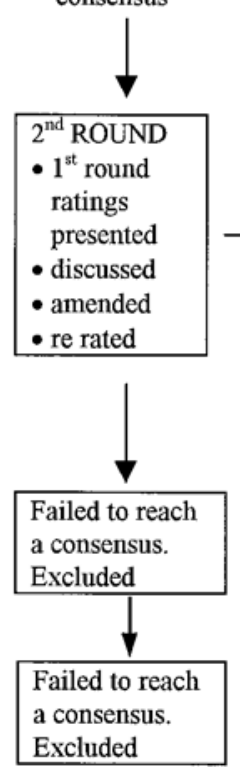

Figure 1

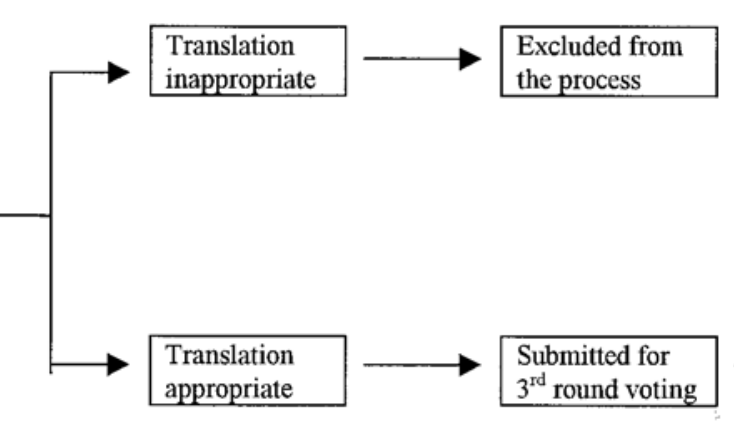

5. The ratings were tabulated and presented. Panellists were presented with a summary of the first round ratings. These included the median and range (dispersion) but not individual ratings. ${ }^{19}$

6. Translations achieving consensus as appropriate, based on the pre-defined guidelines (Table 1) were submitted to the third and final round. ${ }^{16,17,20}$ Translations achieving consensus as inappropriate were excluded from further consideration.

7. Translations failing to achieve consensus were submitted for discussion in the second round when amendments were made, translations re-grouped if necessary and new ratings made. Participants were also given a choice to re-rate the translations during the second round, even if amendments were not made to the translation. It was made clear that the participants need not conform to the group view. ${ }^{17}$
8. The second round ratings were than tabulated and presented to assess the agreements/disagreements. Translations achieving consensus as appropriate were submitted to the third and final round in order to select the one with the highest preferences. Translations achieving consensus as inappropriate were excluded from further consideration. Translations failing to achieve consensus were al so excluded from further consideration.

9. Translations agreed upon as appropriate during the first and second rounds were then re-listed, discussed and ranked to find the most satisfactory translation based on the consensus of the group. Ranking was done by individual confidential voting on a ranking sheet. The level of consensus required in the third round voting was decided in advance ${ }^{16}$ as more than $50 \%$ support from the panel.

10. All the above steps were repeated with each stem of the questionnaire. 
Table 1 Guidelines for rating and definition on agreement

- If fully agree on the meaning conveyed by translation, then rate 9

- If totally disagree on the meaning conveyed by translation, then rate 1

- If the translation carries the same meaning or is fairly satisfactory but requires changing some words, then rate between 5 and 8 depending on the appropriateness otherwise

- If you feel that the translation does not carry the same meaning or is not satisfactory but some of the words used in the translation are useful, then rate between 2 and 5

Agreement (Consensus) ${ }^{19,17}$

Rating 1-3 Agree that the translation is inappropriate

Rating 7-9 Agree that the translation is appropriate

Equivocal

Rating 4-6 $6^{19}$

Disagreement ${ }^{20}$

After discarding the highest and lowest ratings or eliminating two ratings furthest from the median

- If at least one rating fell in 1-3 range when the others' range was at the other extreme

- If at least one rating fell in 7-9 range when the others' range was at the other extreme

In our work, both equivocal and disagreement were considered as failing to reach a consensus

Stage 2

Step 1 We used another qualitative method - a qualitative interview with 25 mothers of twins. $^{21}$ The three stems of the translated version of the questionnaire were used as a semi-structured interview. The responses were noted verbatim, and categorised and analysed later.

Step 2 All three questions with their responses were presented as fixed choice questions and responses. The choice of answers was recorded.

Step 3 The categorised responses elicited through the qualitative interview during Step 1 were compared with the responses obtained during Step 2. Steps 1 and 2 were used as a problem technique and pre-testing. ${ }^{12}$

\section{Stage 3 (bilingual appraisal)}

Seventeen bilingual parents, one from each twin pair, answered the original and the translated questionnaire 3 days apart. This was carried out to measure the degree of similarity in the responses between the two versions. Validity and reliability of the Sinhala version was examined by this procedure.

\section{Results}

Stage 1 (nominal group)

The 'process outcome' of the translation was similar to that reported by Sumathipala and Murray. ${ }^{15}$ However, one of the three question stems, 'Like two peas in a pod' posed a novel challenge. It needed a culturally meaningful translation because it is an idiom. One phrase proposed by a panellist ('eka valle poll' in Sinhala) could be back-translated as 'like coconuts of the same bunch'. Although it conveyed a meaning closer to the original (semantic validity), the group decided against it. The reason was its idiomatic meaning did not fit the original content and construct. This idiom in Sinhala is used with somewhat negative connotation to introduce people with similar attitudes and character rather than the physical resemblance ("people in the same boat'). The al ternative suggested by another panellist ('kapapu palua wage' in Sinhala) could be backtranslated as 'like the split half of'. The significance of this idiomatic phrase is its usage by lay Sinhalese to indicate similarity between two persons. Therefore the group agreed it was the best culturally meaningful, idiomatic phrase that represents the semantic equivalent of 'the peas of the pods'.

\section{Stage 2}

Question 1 The stem of the original questionnaire was: Were your twins 'as like as two peas in a pod'. The Sinhal a equival ent back-translated by the group is, 'Were your twins so similar and difficult to differentiate one from the other, as if one was the split half of the other.' The response to this openended translated stem was compared with the response to the one out of three choices (see Table 2).

Answers of 'yes' to the open-ended question, endorsing that the twins were like the split half of the other, had a perfect correlation (kappa1, Cl 1-1) with the first choice ('yes') in the multiple-choice questionnaire (MCQ). Similarly open-ended answers to this question and the second and third response choices of the MCQ had a modest correlation with kappa values of $0.48(\mathrm{Cl} 0.17-0.79)$ and $0.57(\mathrm{Cl}$ $0.27-0.87)$. This reduced correlation for choices 2 and 3 resulted because the 'no' answers to the openended question were split between choices 2 and 3 . Therefore, the discriminatory power of the open- 
Table 2 Comparison of answers to the first open-ended question and multiple-choice question (MCQ)

\begin{tabular}{|c|c|c|c|c|}
\hline \multirow[b]{2}{*}{ Reg No } & \multirow{2}{*}{$\begin{array}{l}\text { Were your twin children 'as like as } \\
\text { two peas in a pod'? } \\
\text { Response to the open-ended stem }\end{array}$} & \multicolumn{3}{|c|}{$\begin{array}{c}\text { Were your twin children 'as like as two peas in a pod'? } \\
\text { Choice of answers }\end{array}$} \\
\hline & & 1. As like as two peas in a pod & 2. Usual sibling similarity & 3. Quite different \\
\hline 648 & No & & $X$ & \\
\hline 285 & Yes & $\mathrm{X}$ & & \\
\hline 1155 & Yes, identical & $\mathrm{X}$ & & \\
\hline 1232 & Yes & $\mathrm{X}$ & & \\
\hline 1659 & No mother could reply & & & $X$ \\
\hline 1811 & Yes, identical & $\mathrm{X}$ & & \\
\hline 3020 & No, has a slight difference & & $\mathrm{X}$ & \\
\hline 3315 & Yes, like that & $\mathrm{X}$ & & \\
\hline 3788 & Yes, a lot of similarity & $X$ & & \\
\hline 42 & Yes, alike & $X$ & & \\
\hline 1791 & A lot alike & $X$ & & \\
\hline 3177 & Looked alike, had no difference at all & $x$ & & \\
\hline 3743 & No & & & $X$ \\
\hline 4003 & No & & & $X$ \\
\hline 4205 & Yes, no noticeable difference & $X$ & & \\
\hline 4002 & No, elder one thin, other fat & & $X$ & \\
\hline 4209 & No & & $X$ & \\
\hline 465 & No & & & $X$ \\
\hline 4208 & Yes, identical & $X$ & & \\
\hline 2840 & Yes & $X$ & & \\
\hline 4288 & No & & & $X$ \\
\hline 641 & Yes, features similar & $X$ & & \\
\hline 2810 & Yes, even father cannot recognise & $X$ & & \\
\hline 1703 & No & & & $\mathrm{X}$ \\
\hline 1719 & No & & $X$ & \\
\hline
\end{tabular}

ended questionnaire and choice1 of the MCQ are same. However, the discriminatory power of the MCQ choices 2 and 3 (namely 'usual sibling similarity' and 'quite different') was higher than for the open-ended question.

Question 2 The stem of the original questionnaire was 'Were they mixed up at that age? Backtranslation of this was the same. The responses to this open-ended stem of the translation were compared with responses to the three choices of the MCQ (see Table 3).

Open-ended answers to this question showed a Iow correlation to choice 1, with kappa values of 0.23 (Cl 0.05-0.51), a modest correlation to choice 2 with a kappa of $0.59(\mathrm{Cl} 0.27-0.91)$ and a strong correlation to choice 3 with a kappa of $0.76(\mathrm{Cl}$ $0.52-1.01$ ). The low and modest correlation to choices 1 and 2 resulted because the 'Yes' answers to the open-ended question were split between the choices: 1 ('very often') and 2 ('now and then'). The discriminatory power of the 'yes, very often' and 'now and then' is higher in the MCQ. The discriminatory powers of an open-ended questionnaire eliciting 'no' is high, but the responses were incomplete and vague with several connotations. The MCQ makes the discrimination more specific and categorical by the use of the word 'never'.
Question 3 The stem of the original questionnaire was 'By whom they were mixed up?. Back-translation of this was the same. The responses to this openended translated stem were compared with the responses to the three choices (see Table4).

In the analysis, we dichotomised the choices in the MCQ by incorporating choices 1,2 and 3 (parents/relatives/others) into one category and choice 4 (by nobody) into the other. Then we compared these two with the responses to the open-ended questionnaire. We observed that four parents had chosen more than one response to the choices given, instead of picking the most suitable one. They probably forgot the instructions given at the beginning of the questionnaire to select only one of the choices. Therefore, it is important to repeat the instructions with every question. From the above findings, we can safely conclude that the translated version of the questionnaire (MCQ) is a reasonable adaptation of the original questionnaire.

\section{Stage 3}

During this stage, the adapted questionnaire was compared with the original by using 17 bilingual parents of twins. These 17 parents were a different group from the 25 parents who participated in Stage 2. They were given the original English questionnaire to mark their responses. Three days later, 
Table 3 Comparison of answers to the second open-ended question with answers selected from the MCQ

\begin{tabular}{|c|c|c|c|c|}
\hline Reg No & $\begin{array}{l}\text { Were they mixed up at that age? } \\
\text { Response to the open-ended stem }\end{array}$ & \multicolumn{2}{|c|}{ Were they mixed up at that age? } & Never \\
\hline 648 & No & & & $\mathrm{x}$ \\
\hline 285 & Yes & & $\mathrm{x}$ & \\
\hline 1155 & Yes, very often & $\mathrm{x}$ & & \\
\hline 1232 & Yes & & $\mathrm{x}$ & \\
\hline 1659 & No & & & $\mathrm{x}$ \\
\hline 1811 & Yes, to some extent & $\mathrm{x}$ & $\mathrm{x}$ & \\
\hline 3020 & Not by me & & $\mathrm{x}$ & \\
\hline 3315 & Yes, at times & & $\mathrm{x}$ & \\
\hline 3788 & No & & $\mathrm{x}$ & \\
\hline 42 & Usually not by me, but at times & & $\mathrm{x}$ & \\
\hline 1791 & Yes, so much had to use a scar on ear & & $\mathrm{x}$ & \\
\hline 3177 & No, had a small difference & & & $\mathrm{x}$ \\
\hline 3743 & No, has noticeable difference & & & $\mathrm{x}$ \\
\hline 4003 & Yes, at times & & $\mathrm{x}$ & \\
\hline 4205 & Yes & & $\mathrm{x}$ & \\
\hline 4002 & No & & & $\mathrm{x}$ \\
\hline 4209 & No & & & $\mathrm{x}$ \\
\hline 465 & No & & & $\mathrm{x}$ \\
\hline 4208 & No, one had a chubby face, if not same & & $\mathrm{x}$ & \\
\hline 2840 & Did not mix up & & & $\mathrm{x}$ \\
\hline 4288 & No, one is tall, the other short & & & $\mathrm{x}$ \\
\hline 641 & Yes, I got mixed up at times & & $\mathrm{x}$ & \\
\hline 2810 & No, not by me & & & $\mathrm{x}$ \\
\hline 1703 & No, not a bit & & & $\mathrm{x}$ \\
\hline 1719 & No, not by me & & & $x$ \\
\hline
\end{tabular}

Table 4 Comparison of answers to third open-ended question and answers selected from the MCQ

\begin{tabular}{|c|c|c|c|c|c|}
\hline \multirow[b]{2}{*}{ Reg No } & \multirow{2}{*}{$\begin{array}{l}\text { Who mixed them up? } \\
\text { Response to the open-ended stem }\end{array}$} & \multirow[b]{2}{*}{ Parents } & \multicolumn{3}{|c|}{$\begin{array}{l}\text { Who mixed them up? } \\
\text { Choice of answers }\end{array}$} \\
\hline & & & Relatives or neighbours & Others & Nobody \\
\hline 648 & No-one & & & & $x$ \\
\hline 285 & Parents & $\mathrm{X}$ & & & \\
\hline 1155 & Teacher cannot still make out & $\mathrm{x}$ & $\mathrm{X}$ & & \\
\hline 1232 & Mother, particularly when dark & $\mathrm{X}$ & $\mathrm{x}$ & $\mathrm{X}$ & \\
\hline 1659 & Grandmother & & & $\mathrm{X}$ (teacher) & \\
\hline 1811 & Outsiders & $\mathrm{X}$ & $\mathrm{x}$ & & \\
\hline 3020 & Child minder & & $\mathrm{x}$ & & \\
\hline 3315 & Outsiders, but I can & & $\mathrm{x}$ & & \\
\hline 3788 & Rel atives and others & & $\mathrm{x}$ & & \\
\hline 42 & Every one, even father and outsiders & & $\mathrm{x}$ & & \\
\hline 1791 & Most people would & & $\mathrm{X}$ & & \\
\hline 3177 & No & & & & $\mathrm{X}$ \\
\hline 3743 & No, people who came across them can & & $\mathrm{x}$ & & \\
\hline 4003 & 2 Contradictory remarks made & $\mathrm{X}$ & & & \\
\hline 4205 & Parents, siblings, outsiders & & $\mathrm{X}$ & & \\
\hline 4002 & People who rarely see them & & & $\mathrm{X}$ & \\
\hline 4209 & No, just like normal siblings & & & $\mathrm{x}$ & \\
\hline 465 & No-one mixed up & & & & $\mathrm{X}$ \\
\hline 4208 & Father and others at times & & $\mathrm{x}$ & & \\
\hline 2840 & Relatives and people in the village & & $\mathrm{x}$ & & \\
\hline 4288 & No, any one can recognise & & $x$ & & \\
\hline 641 & Father and others in the family & $\mathrm{X}$ & $\mathrm{x}$ & $\mathrm{X}$ & \\
\hline 2810 & Relatives and neighbours & & $\mathrm{x}$ & & \\
\hline 1703 & Did not mix up & & & & $\mathrm{X}$ \\
\hline 1719 & People say similar & & & $\mathrm{x}$ & \\
\hline
\end{tabular}




\section{Discussion}

Adequacy of a diagnostic instrument in a given culture does not guarantee its validity in another (even given a faithful translation) especially when the cultures are considerably different. ${ }^{8}$ Culturally sensitive research involves a careful and thorough adaptation of the instrument to be studied. ${ }^{8}$

In Stage 1, the use of a nominal group for translation and consensus generation was another example of how an individual translation could differ from a group effort. This reconfirms the earlier work by Sumathipala and Murray ${ }^{15}$ that a qualitative method (nominal group/expert panel to translate and assess the extent of agreement (consensus measurement) on the appropriateness of the translation and resolve disagreement (consensus development)) has advantages.

The stem of the first question, an idiom, posed a novel challenge as it demanded a culturally meaningful translation. One of the translations conveyed a meaning closer to the original (semantic validity), but its idiomatic meaning did not fit the original content and construct. This idiom in Sinhala is used with a negative connotation to introduce people with similar attitudes and character rather than the physical resemblance. The group decided on an alternative as the best culturally meaningful, idiomatic phrase equival ent of 'the peas of the pods' as it indicated similarity between two persons. In this process the group did not translate but discovered an idiom al ready in use by Sinhal ese to suit the original idiom. This was beyond a mere translation.

Stage 2 revealed that the verbatim responses recorded during the qualitative interview and the responses to the forced-choice answers to the full questionnaire were mutually inclusive and exhaustive. Stage3, bilingual appraisal, revealed a perfect agreement between the two questionnaires with a kappa of 1 . Both the resource and adapted questionnaires showed that $15(60 \%)$ of the 25 twin pairs are $\mathrm{MZ}$ and $10(40 \%)$ are $\mathrm{DZ}$.

Stages 2 and 3 established that the adaptation process followed in this work has produced a comparable and valid instrument to the source questionnaire, and also a culturally suitable construct. Therefore we conclude that adaptation and preliminary validation is satisfactory. In an ideal situation the adapted version should be crosschecked against the gold standard, ie genetic markers. When it is not available or not easily accessible, as in the developing world, there should be alternative ways of reaching the best possible approximation. We used a combination of qualitative methods to reach that need. This attempt is only a preliminary validation. Zygosity determination by genetic mark- ers should now be compared with this questionnaire to establish the definitive validation.

\section{Acknowledgements}

The authors are grateful to the participants in the nominal group - Dr A Padeniya, Dr V Dissanayake, Dr $\mathrm{P}$ Katulanda, Dr RP Abeywickrama, $\mathrm{Dr} \mathrm{AH}$ Priyani, Dr R Wijesuriya, Miss NML Radhika, K Wijesingha and $\mathrm{S}$ Hewege.

\section{References}

1 Nichols PC, Bilbro WC. The diagnosis of twin zygosity. Acta Genet Stat Med 1966; 16: 265-275.

2 Jablon S, Neel N, Gershowitz H, Atkinson GF. The NAS-NRC twin panel: Methods of the construction of the panel, zygosity diagnosis and proposed use. Am J Hum Genet 1967; 19: 133-161.

3 Cederl of R, Friberg L, Jonsson E, Kaij L. Studies on similarity diagnosis in twins with the aid of mailed questionnaires. Acta Genet Stat Med 1961; 11: 338-362.

4 Sarna S, Kaprio J, Sistonen P, Koekenvuo M. Diagnosis of twin zygosity by mailed questionnaire. Hum Hered 1978; 28: 241-254.

5 Goldsmith $\mathrm{HH}$. A zygosity questionnaire for young twins: a research note. Behav Genet 1991; 21: 257-269.

6 Ooki S, Yamada K, Asaka A. Zygosity diagnosis of twins by questionnaire for twins' mothers. Acta Gen Med Gemellol 1993; 42: 17-22.

7 Sumathipala A, Fernando DJS, Siribaddana SH, Abeysingha MRN, Jayasekera RW, Dissanayake VHW, De Silva N. Establishment of a National Twin Registry in Sri Lanka. Twin Res 2000; 3: 202-204.

8 Bravo M, Canino GJ, Rubio-Stipec M, Woodbuyr-Farina M. A cross-cultural adaptation of a psychiatric epidemiological instrument. The diagnostic interview schedules adaptation in Puerto Rico. Cul Med Psychiatry 1991; 15: 1-18.

9 Kleinman A. Anthropology and psychiatry. The role of culture in cross-cultural research on illness. Br JPsychiatry 1987; 151: 447-454

10 Gaviria M, Pathak DS, Michell T, Flaherty A, Winthrop RM, Martinez H, Pacheco GC, Richman J, Birz S. Designing and Adapting Instruments for a Cross-cultural Study on Immigration and Mental Health in Peru. American Psychiatric Association: 1984.

11 Bravo M, Woodbuyr-Farina M, Canino J, Rubio-Stipec M. The Spanish translation and cultural adaptation of the diagnostic interview schedule in Puerto Rico. Cul Med Psychiatry 1993; 17:329-344.

12 Guilliman F, Bombardier C, Beaton D. Cross cultural adaptation of health related quality of life measures: Literature review of proposed guidelines. J Clin Epidemiol 1993; 46: 1417-1432.

13 Drennan G, Levett A, Swarts L. Hidden dimension of power and resistance in the translation process: A South African study. Cul Med Psychiatry 1991; 15: 361-381.

14 Brislin, Loner, Thorndike. Cross Cultural Research Methods. John Wiley and Sons: New York 1973. 
15 Sumathipala A, Murray J. New approach to translating instruments for cross-cultural research: A combined qualitative and quantitative approach for translation and consensus generation. Int J Meth Psychiatr Res 2000; 9:87-95.

16 Fink $A$, Kosecoff J, Chassin $M$ et al. Consensus methods: Characteristics and Guideline for use. Am J Public Health 1984; 74:979-983.

17 Jones J, Hunter D. Consensus methods for medical and health services research. BMJ 1995; 311:376-380.

18 Scott EA, Black NA. When does consensus exist in expert panels? J Public Health Med 1991; 13:35-39.
19 Hunter DJW, McKie CM, Sanderson CFB, Black NA. Appropriate indications for prostetectomy in the UK. Results of a consensus panel. J Epidemiol Commun Health1994; 48: 58-64.

20 Scott EA, Black NA. Appropriateness of cholesystectomy - a consensus panel approach. Gut 1991; 9: 1066-1070.

21 Pope C, Mays N. Reaching the parts other methods cannot reach: an introduction to qualitative methods in health and health services research. BMJ 1995; 311:42-45. 